\title{
The Spliceosomal Component Sf3b1 is Essential for Hematopoietic Differentiation in Zebrafish
}

\author{
Adriana De La Garza ${ }^{\# 1,2}$, Rosannah C. Cameron ${ }^{\# 1,2}$, Sara Nik ${ }^{1,2}$, Sara G. Payne ${ }^{1,2}$, and \\ Teresa V. Bowman $\#, 1,2,3$ \\ ${ }^{1}$ Department of Developmental and Molecular Biology, Albert Einstein College of Medicine \\ ${ }^{2}$ Gottesman Institute for Stem Cell Biology and Regenerative Medicine, Albert Einstein College of \\ Medicine \\ ${ }^{3}$ Department of Medicine (Onocology), Albert Einstein College of Medicine \\ \# These authors contributed equally to this work.
}

\section{Abstract}

SF3B1 (Splicing factor 3b, subunit 1) is one of the most commonly mutated factors in myelodysplastic syndrome (MDS). Although the genetic correlation between SF3B1 mutations and MDS etiology are quite strong, no in vivo model currently exists to explore how SF3B1 loss alters blood cell development. Using zebrafish mutants, we show that proper function of Sf3b1 is required for all hematopoietic lineages. Similar to MDS patients, zebrafish $s f 3 b 1$ mutants develop a macrocytic anemia-like phenotype due to a block in maturation at a late progenitor stage. The mutant embryos also develop neutropenia as their primitive myeloid cells fail to mature and turn on differentiation markers such as 1-plastin and myeloperoxidase. In contrast, production of definitive hematopoietic stem and progenitor cells (HSPCs) from hemogenic endothelial cells within the dorsal aorta is greatly diminished, while arterial endothelial cells are correctly fated. Notch signaling, imperative for the endothelial-to-hematopoietic transition, is also normal, indicating HSPC induction is blocked in $s f 3 b 1$ mutants downstream or independent of Notch signaling. The data demonstrate $\mathrm{Sf} 3 \mathrm{~b} 1$ function is necessary during key differentiation fate decisions in multiple blood cell types. Zebrafish $s f 3 b 1$ mutants offer a novel animal model to explore the role of splicing in hematopoietic development and provide an excellent in vivo system to delve into the why and how Sf3b1 dysfunction is detrimental to hematopoietic differentiation, which could enlighten MDS diagnosis and treatment.

\footnotetext{
\#To whom correspondence should be addressed Address: 1300 Morris Park Avenue, Bronx, NY 10461, teresa.bowman@einstein.yu.edu, phone- (718) 430-4001, fax- (718) 430-8567.

Publisher's Disclaimer: This is a PDF file of an unedited manuscript that has been accepted for publication. As a service to our customers we are providing this early version of the manuscript. The manuscript will undergo copyediting, typesetting, and review of the resulting proof before it is published in its final citable form. Please note that during the production process errors may be discovered which could affect the content, and all legal disclaimers that apply to the journal pertain.

Authorship Contributions

ADG, RCC, SN, SP, and TVB performed experiments and analyzed data. ADG, RCC, and TVB wrote and edited the manuscript.

The authors have no conflicts to disclose.
} 


\section{Introduction}

Transcription and RNA processing are interconnected processes that are essential for regulating gene expression. Genes involved in these processes are commonly mutated in hematological malignancies. Although many key transcription factors involved in both embryonic and adult hematopoiesis have been identified ${ }^{1}$, the role of RNA processing factors is unclear.

Hematopoietic specification occurs in multiple waves. The first or primitive wave of hematopoietic induction consists mainly of erythrocytes and myeloid cells that sustain the embryo until adult cells are formed ${ }^{2}$. The definitive wave, which occurs later in development, generates multipotent progenitors and hematopoietic stem and progenitor cells (HSPCs) that give rise to all the mature blood cells required throughout the lifetime of an organism $^{3}$. The first HSPCs emerge from hemogenic endothelium in the ventral wall of the dorsal aorta and then colonize secondary organs, such as fetal liver, placenta, thymus and bone marrow in mammals and the caudal hematopoietic tissue, thymus, and kidney marrow in zebrafish (reviewed in $^{1-3}$ ). Much is known about the transcription factors, such as runx1 (runt-related transcription factor 1), gata2, scl (stem cell leukemia), gfil (growth factor inducible-1), pu.1, and gata1 among others, that orchestrate developmental hematopoietic specification, but less is known about the role of RNA processing.

Splicing of pre-mRNA is a co-transcriptional event that diversifies the proteome and contributes to cell fate control. The spliceosome is a macromolecular machine comprised of five snRNPs (small nuclear ribonucleoproteins), each made of large, multicomponent protein complexes and snRNAs (small nuclear RNAs). Recent genome sequencing efforts identified mutations in several spliceosomal components in hematologic malignancies, such as acute myeloid leukemia and myelodysplastic syndrome (MDS) ${ }^{4-8}$. These clinical data suggest splicing regulation is important in hematopoiesis. Mutations in spliceosomal components are mutually exclusive in MDS, yet mutations in each factor correlate with a different disease phenotype. For example, mutations in the U2 snRNP component SF3B1 (Splicing factor 3b, subunit 1) show a strong correlation with subtypes of MDS with refractory anemia and ringed sideroblasts ${ }^{9}$. In contrast, mutations in the splicing accessory protein SRSF2 (Serine/ arginine-rich splicing factor 2) or U2AF1 (U2 snRNP-associated factor-1) correlate with poor prognosis and are found more frequently in more malignant forms of MDS, such as refractory anemia with excess blasts ${ }^{4-8,10}$. These clinical data imply that splicing factors might employ different mechanisms and/or have different cell-type selective tropisms that lead to distinct MDS features. Thus, it is imperative to understand the specific roles of each splicing subunit.

In support of these clinical observations, zebrafish spliceosomal mutants display some overlapping and distinct defects in hematopoiesis ${ }^{11-16}$. For example, mutants in the RNA helicases $d d x 18^{h i 1727}$ (DEAD-box protein 18) and $d h x 8^{\text {hg9 }}$ (DEAH-box protein 8) have greatly reduced primitive myeloid and erythroid cells, while $d d x 4 b^{\text {hi2137 }}$ (DEAD-box protein 46) mutants have impaired primitive erythropoiesis as well as defects in definitive HSPCs maintenance ${ }^{11-13}$. In contrast, mutants in the U5 snRNP component prpf\&gll (pre$m R N A$ processing factor 8 ), have defective myeloid lineage development, but no defects in 
erythropoiesis or definitive HSPC induction ${ }^{14}$. The U2 snRNP-associated factor U2af1 is also critical for hematopoietic development, as zebrafish $u 2 a f 1^{\text {hil }}{ }^{\text {g9 }}$ mutants have diminished erythroid differentiation and fewer HSPCs ${ }^{16}$. In contrast, mutants for the U1 snRNP component snrnp70hi3018 (small nuclear ribonucleoprotein, 70kDa) and the U2 snRNP member sf3a3hi1950 (splicing factor 3A, subunit 3) show diminished HSPC formation, but no defects in primitive hematopoiesis ${ }^{15}$. These data show that dysfunction in numerous components of the spliceosome negatively impact hematopoiesis, but also demonstrates differential cell-type sensitivity depending on the spliceosomal constituent mutated.

$S F 3 B 1$ is the most commonly mutated spliceosomal component in $\mathrm{MDS}^{4-10}$, yet its in vivo consequences on developmental hematopoiesis are unknown. Homozygous Sf3b1-null mice are embryonic lethal pre-implantation, precluding analysis of development ${ }^{17}$. Adult heterozygous $S f 3 b 1$ mutants develop macrocytic anemia and have HSC self-renewal defects, demonstrating cell-type selective susceptibilities to $S f 3 b 1$ dosage ${ }^{18-20}$. To explore the in vivo outcomes of $s f 3 b 1$ depletion on developmental hematopoiesis, we characterized a recently described zebrafish $s f 3 b 1$ mutant $^{21,22}$. These mutants contain a loss-of-function mutation that is partially compensated by retention of maternally-deposited wild-type protein and mRNA $^{21}$. The $s f 3 b 1$ mutants show increased neuronal cell death, similar to many other spliceosomal mutants, and have defects in neural crest cell induction ${ }^{21}$. Using the $s f 3 b 1^{\text {hi33 } 34 a}$ loss-of-function allele, we defined an essential role for $s f 3 b 1$ in blood development. The primitive erythroid and myeloid cells are specified normally, but then fail to mature. In the definitive wave, HSPCs do not form, although the precursor endothelial cells are present. Notch signaling, a critical mediator of hemogenic endothelium and HSPC induction, also appears unaffected in the dorsal aorta in $s f 3 b 1$ mutants. These data indicate $s f 3 b 1$ is required during hemogenic specification downstream or independent of Notch. Overall, our findings show $s f 3 b 1$ function is critical for key hematopoietic cell fate decisions during early development and establish $s f 3 b 1$ zebrafish mutants as an in vivo model to delineate how Sf3b1 dysfunction alters hematopoietic decisions, which could impact MDS diagnosis and treatment.

\section{Materials and methods}

\section{Zebrafish}

Zebrafish were maintained as described. ${ }^{23,24}$ All fish were maintained according to IACUC approved protocols in accordance with Albert Einstein College of Medicine research guidelines.

\section{Zebrafish lines used}

The $s f 3 b 1$ mutant line hi3394a contains a viral insertion between the $1^{\text {st }}$ and $2^{\text {nd }}$ exons of the gene $s f 3 b 1$, resulting in a premature stop codon in exon $2^{21,22}$. Wild-type and heterozygous embryos are phenotypically indistinguishable from one another, thus for all of the experiments presented in the paper, wild-type and $s f 3 b 1$ heterozygous embryos are cumulatively referred to as wild type. As $s f 3 b 1$ homozygous mutants are developmentally delayed and show defects in melanocyte development ${ }^{21}$, mutant embryos were age-matched for all analyses according to morphological features. In addition, analyses of each 
hematopoietic population were done for at least two developmental time points to control for any variation in stage matching. Mutant embryos die between 2-3dpf, precluding analysis of later stages ${ }^{21}$. For fluorescent imaging and flow cytometry analysis, we crossed sf $3 b 1^{\text {hi3 } 3394 a}$ heterozygous animals to the following transgenic lines: $\operatorname{Tg}(\text { gatal:gfp })^{25}, \operatorname{Tg}(\text { kdrl:dsRed })^{26}$, $T g$ (cmyb:gfp $)^{27}, \operatorname{Tg}(\text { lyz:dsRed })^{\text {nz50 } 28}$ and $\operatorname{Tg}\left(\right.$ tplbglob:eGFP) $(\text { tp1:eGFP })^{29}$.

\section{Morpholino injections}

To knockdown $s f 3 b 1$ levels, we utilized a published translation-inhibiting morpholino ${ }^{21}$. Wild-type embryos were injected with $0.1 \mathrm{ng}(0.5 \mathrm{nl}$ of $0.2 \mu \mathrm{g} / \mu \mathrm{l}$ stock) and compared to noninjected sibling controls.

\section{Whole-mount in situ hybridization}

In situ hybridization steps were performed as described previously by Thisse et al..$^{30}$ with minor modifications: before proteinase K permeabilization, embryos older than 28 hours post fertilization (hpf) were bleached after re-hydration to remove pigmentation. The bleaching was done for 5-10 minutes using a bleaching solution of $0.8 \% \mathrm{KOH}, 0.9 \% \mathrm{H}_{2} \mathrm{O}_{2}$ and $0.1 \%$ Tween 20. The following probes were used: $\beta$-globin ${ }^{31}, e p h r i n b 2^{32}, f l t 4^{32}, k d r \beta^{3}$, 1-plastin ${ }^{34}$, mpx $^{35}$, notch $1 b^{32}$, notch $3^{32}$, pu. $1^{36}$, runx $1^{37}$, gata $2 b^{38}$ and $s c \beta^{39}$. Embryos were then scored manually, imaged and genotyped. Separate PCR reactions were used for detecting wild-type and mutant $s f 3 b 1$ alleles. A common reverse primer $\left(5^{\prime}\right.$ -

GACAATCACCCACGGCCATG-3') was paired with unique forward primers for the wildtype (5'-GATCGCCAAAACACATGATG-3 $\left.{ }^{\prime}\right)$ or mutant alleles $\left(5^{\prime}\right.$ CTGTCCATCTGTTCCTGAC-3').

\section{Flow cytometry}

For generation of single cell suspensions, 10-20 embryos were first removed from their chorions using pronase (Roche), and then homogenized by manual dissociation using a sterile razor blade followed by digestion with Liberase (Roche). For the digestion, dissociated embryos were resuspended in $3 \mathrm{ml} 1 \times$ Dulbeccos-PBS (D-PBS) (Life Technologies) supplemented with a 1:65 dilution $(46 \mu \mathrm{l})$ of $5 \mathrm{mg} / \mathrm{ml}$ Liberase and then incubated at $37^{\circ} \mathrm{C}$ for 8 minutes. The reaction was s topped with $5 \%$ (157 $\left.\mu \mathrm{l}\right)$ fetal bovine serum (FBS) (Life Technologies). The cells were then filtered twice through 40- $\mu \mathrm{m}$ cell strainers (Falcon) and pelleted by centrifugation at $3000 \mathrm{rpm}$ for 5 minutes. Cell pellets were resuspended in 1-2 ml FACS buffer (0.9× D-PBS, 5\% FBS, $1 \%$ Penn/Strep (Life Technologies)). DAPI (4',6-diamidino-2-phenylindole) was added to a final concentration of $1 \mu \mathrm{g} / \mathrm{ml}$ to facilitate exclusion of dead cells from the analysis. Samples were analyzed with a LSRII flow cytometer (BD Biosciences) and FlowJo version 10.0.8.

\section{Fluorescent imaging}

Embryos (24-36 hpf) were anesthetized with tricaine and oriented in a drop of 3\% wt/vol methylcellulose and then mounted in 1\% agarose in 35-mm imaging dishes (MatTek) as previously described ${ }^{40}$. Fluorescent imaging was performed with a Zeiss Axio Observer A1 with an AxioCam ICM1 camera and Zeiss Zen 2 software. Fluorescence was detected with GFP and Texas-Red filters. 


\section{May-Grunwald Giemsa staining of primitive erythroid cells}

Two-day old embryos were placed on poly-L-lysine coated slides in a drop of $1 \times$ D-PBS $+1 \%$ bovine serum albumin (Sigma). Blood cells were released from the embryo by puncturing the pericardial sac and upper yolk sac with fine forceps. The slides were air-dried at room temperature prior to staining. For staining, slides were immersed in undiluted MayGrunwald stain (Eng Scientific May-Grunwald stain solution 1, Fisher Scientific) for 2 minutes and briefly rinsed in $\mathrm{ddH}_{2} \mathrm{O}$. Slides were then immersed in dilulted Giemsa stain (diluted 1:4 with milliQ water) for 20 minutes (Eng Scientific May-Grunwald stain solution II, Fisher Scientific) and briefly rinsed in $\mathrm{ddH}_{2} 0$. Once slides were dry, a drop of Permount solution (Fisher Scientific) was added and slides were covered with cover slips and left overnight to dry. Once slides were dry, the cells were visualized with a $63 \times$ oil-immersion lens.

\section{O-dianisidine and apoptotic marker analysis}

O-dianisidine staining was performed as described previously ${ }^{35}$. Dechorionated, live embryos were soaked in o-dianisidine staining solution $(0.62 \mathrm{mg} / \mathrm{ml}$ o-dianisidine (Sigma), $10.9 \mu \mathrm{M}$ sodium acetate, and $0.65 \% \mathrm{H}_{2} \mathrm{O}_{2}$ ) for $20 \mathrm{~min}$ in the dark. Acridine Orange staining was performed as previously described ${ }^{41}$. Dechorionated, live embryos were soaked in a $2 \mu \mathrm{g} / \mathrm{ml}$ acridine orange (Sigma) solution for one hour in the dark. Immunofluorescence analysis was performed for active caspase 3 (BD Biosciences) at $24 \mathrm{hpf}$ as previously described $^{42}$.

\section{Treatment with SNAP}

Embryos from an incross of $s f 3 b 1$ mutants were treated with the Nitric Oxide (NO) donor SNAP (S-Nitroso-N-Acetyl-D,L-Penicillamine) from $7 \mathrm{hpf}$ until $28 \mathrm{hpf}$. Doses tested were based on previous studies ${ }^{43}$ and included $10 \mu \mathrm{M}$ and $30 \mu \mathrm{M}$. Control embryos were treated with $0.06 \%$ DMSO as a vehicle control.

\section{Statistics}

For pairwise comparisons, the Student's t-test (unpaired, two-tailed) was employed. For comparison of population distributions, Chi-squared analysis was performed. Statistical test utilized is designated in the figure legend for each analysis.

\section{Results}

\section{Erythroid maturation is hindered in sf $3 b 1$ mutants}

To characterize the blood compartments in $s f 3 b 1$ zebrafish, we first assessed primitive hematopoietic induction. Primitive erythroid and myeloid cells arise from $s c l$-positive mesoderm ${ }^{3,39}$. We observed no difference in $s c l$ expression between $s f 3 b 1$ mutants and wildtype siblings at the 12 somite stage (ss)/14hpf (Figure 1A). Gata1 is an erythroid-specific transcription factor that is essential for erythropoiesis ${ }^{44}$, thus to determine if erythroid cell identity was properly specified in $s f 3 b 1$ mutants, we performed flow cytometry analysis of sf3b1 Tg (gata1:eGFP) embryos at $24 \mathrm{hpf}$. We observed a subtle, but significantly higher percentage of gata1 positive cells in $s f 3 b 1$ mutants compared to wild-type siblings (Figure 
1B-D). At $48 \mathrm{hpf}$ the number of gata1-positive hematopoietic cells remained high in mutants, but was reduced in wild-type siblings (Figure 1E). The reduction in gata1-positive cells in wild-type embryos is consistent with previous studies that showed a down-regulation of gata1 mRNA at 36-48 hpf thought to be associated with erythroid differentiation or maturation $^{45,46}$. The perdurance of high gatal-expressing cells in $s f 3 b 1$ mutants might be a reflection of a block in differentiation or maturation.

To investigate the differentiation status of erythroid cells in sf $3 b 1$ mutants, we examined the expression of the differentiation marker $\beta$-globin by in situ hybridization. Expression of $\beta$ globin, a component of oxygen-carrying hemoglobin, was normal in sf $3 b 1$ mutants at $24 \mathrm{hpf}$ (Figure 1F). Functional hemoglobin can be detected in wild-type red blood cells beginning around 36-48 hpf using o-dianisidine staining ${ }^{47}$. In sf3b1 mutants at $36 \mathrm{hpf}$, we observed little o-dianisidine-positive erythroid cells (Figure 1G). Mutants display some developmental delay that becomes more severe as the embryos get older, thus we also looked at odianisidine staining at $48 \mathrm{hpf}$ and noted no improvement to hemoglobin function (Figure 1H). Moreover, the level of o-dianisidine staining in the $48 \mathrm{hpf}$ embryos is lower than wild types at $36 \mathrm{hpf}$ indicating the decrease in o-dianisidine is unlikely solely due to developmental delay. To validate the findings in the mutants, we also tested hemoglobin function in $s f 3 b 1$ morpholino-injected embryos ${ }^{21}$, and noted a similar decrease in odianisidine staining (Figure S1A). To further characterize the defects in primitive erythropoiesis in $s f 3 b 1$ mutants, we analyzed the morphology of isolated red blood cells using May-Grunwald-Giemsa staining (Figure 1I). Mutants had a higher percentage of proerythroblasts and a corresponding decrease in more mature erythroid precursors at $48 \mathrm{hpf}$ (Figure 1J). Furthermore, the later stage orthochromatophilic erythroblasts in the sf $3 b 1$ mutants showed a megaloblastoid-like phenotype compared to wild type (Figure 1I). Together, our results indicate $s f 3 b 1$ mutant erythroid cells have a late-stage block in differentiation.

\section{Myeloid cell development is impaired in sf3b1 mutants}

During development, primitive myelopoiesis originates in both the anterior and posterior lateral plate mesoderm from $s c l$-expressing cells ${ }^{2}$. As mentioned above, $s c l$ expression was unchanged in $s f 3 b 1$ mutants, either in the anterior or posterior blood islands (Figure 1A). The transcription factor pu. 1 is a master regulator of myeloid cell fate and is first expressed in developing zebrafish around $12 \mathrm{hpf} / 6 \mathrm{ss}^{36}$. In $s f 3 b 1$ mutants, pu. 1 -expressing cells are detected at $22 \mathrm{hpf}$, but are slightly diminished and less dispersed across the yolk, suggesting a potential delay in differentiation (Figure 2A). We next examined the expression of markers of mature myeloid cells. Differentiation of myeloid cells is marked by expression of genes such as 1-plastin or myeloperoxidase (mpx), beginning around $16 \mathrm{hpf}$ and $19 \mathrm{hpf}$, respectively. At $24 \mathrm{hpf}$, expression of 1-plastin and $m p x$ are greatly diminished in $s f 3 b 1$ mutants (Figure 2B,C). To rule out a contribution of developmental delay for the lack of mature myeloid marker expression in sf $3 b 1$ mutants, we examined the expression of 1-plastin and $m p x$ at 28 hpf. The markers were still diminished in the mutants, suggesting a block in myeloid cell differentiation (Figure 2D,E). We confirmed the decrease in $m p x$ at $28 \mathrm{hpf}$ in sf3b1 morpholino-injected embryos (Figure S1B). To further test this hypothesis, we used flow cytometry to quantify the frequency of $1 y z: d s R e d$ myeloid cells at $36 \mathrm{hpf}$. Lyz:dsRed 
myeloid cells were over 22-fold decreased in $s f 3 b 1$ mutants compared to wild-type siblings (Figure 2F,G). Erythroid-myeloid progenitors are also arising in zebrafish embryos at this time during development ${ }^{48,49}$. To characterize this cell population, we examined $p u .1$ at 26 hpf and 1-plastin and $m p x$ at $30 \mathrm{hpf}$ within the posterior blood island (PBI) (Figure 3). As in the primitive wave, we observed some pu.1-positive cells in the PBI, but greatly diminished expression of more differentiated markers. Taken together, these results show myeloid specification initiates normally in the mutants, but myeloid cell differentiation is hindered.

\section{HSPC formation is inhibited in sf3b1 mutants}

We next examined if there were changes to definitive HSPC formation. HSPCs emerge from hemogenic endothelial cells that reside along the ventral wall of the dorsal aorta ${ }^{3,50}$. One of the earliest markers of HSPCs is runx $1^{37}$. Expression of runx 1 is nearly absent within the aorta of $28 \mathrm{hpf} s f 3 b 1$ mutants, but is readily detected in wild-type siblings (Figure S2A). Mutant $s f 3 b 1$ embryos have a variable reduction in blood flow and decrease in aortic lumenization, thus we wanted to determine if these defects were the main contributors to the observed HSPC loss in mutants. Although blood flow is needed for HSPC formation, it is dispensable for the early induction of runx 1 expression at $24 \mathrm{hpf}^{51}$. To determine if the decrease in HSPC gene expression is present at this earlier stage, we examined expression of runx 1 and another HSPC marker, gata $2 b^{38}$, at $24 \mathrm{hpf}$. In agreement with the results at 28 hpf, we observed diminished expression of both markers at $24 \mathrm{hpf}$ (Figure 4A, B). We confirmed the decrease in runx 1 expression at $24 \mathrm{hpf}$ in $s f 3 b 1$ morpholino-injected embryos (Figure S1C). Newly-born HSPCs co-express markers of hematopoietic and endothelial cells $^{52}$. This property can be visualized in embryos that are double-transgenic for c-myb:gfp and kdrl:dsred (Figure 4C). We incrossed c-myb:gfp;kdrl:dsred transgenic sf3b1 heterozygotes and quantified HSPC number at $38 \mathrm{hpf}$ in mutant and wild-type siblings. Wild-type embryos had 5.4 c-myb:gfp;kdrl:dsred double positive cells on average, while sf $3 b 1$ mutants had only 1.4 (Figure 4 C,D). Due to the variable decrease in blood flow and aortic lumen size observed in sf3b1 mutants, we quantified c-myb:gfp;kdrl:dsred double positive cells in embryos with different lumen sizes, but noted no significant difference in the number of double positive cells, suggesting the defect in HSPC formation is independent of these defects. To further confirm that the decreased HSPC production was not due to loss of signals from blood flow, we treated embryos with the nitric oxide donor SNAP (SNitroso-N-Acetyl-D,L-Penicillamine), which was previously shown to restore HSPC levels in mutants lacking blood flow ${ }^{43,51}$. Treatment with SNAP had no effect on runx 1 levels in mutant embryos (Figure S2B). Combined, these data demonstrate a severe defect in HSPC formation in $s f 3 b 1$ mutants independent of the effects on blood flow and aortic lumenization.

\section{Aortic specification and Notch signaling are unperturbed in sf3b1 mutants}

Hemogenic endothelial cells in the ventral wall of the dorsal aorta are the immediate precursor to HSPCs, thus proper vasculature formation is an essential prerequisite for HSPC induction ${ }^{50}$. We next analyzed the expression pattern of endothelial markers in $s f 3 b 1$ mutants and wild-type siblings. The pan-endothelial marker $k d r l$ is expressed in the dorsal aorta, cardinal vein, and intersomitic vessels in both wild-type and mutant siblings at 24 and 28 hpf (Figure 5A and S3A). Proper artery-vein identity precedes HSPC induction ${ }^{3,50}$. To 
determine if vessel identity was correctly initiated in $s f 3 b 1$ mutants, we examined artery and vein-specific markers. The vein marker flt 4 shows the presence of a well-formed posterior cardinal vein in 24 and 28 hpf mutant embryos (Figure 5B and S3B). Aorta-specific markers ephrinb2, notch1b, and notch 3 are expressed in the aorta of both wild type and mutants at 24 and $28 \mathrm{hpf}$, demonstrating proper specification of aortic endothelium (Figure 5C and S3C). The Notch pathway gives arterial identity to endothelial cells, and is a critical signal for HSPC specification ${ }^{29,50}$. Correct expression of notch $1 b$ and notch 3 within the dorsal aorta suggests an intact signaling network, but does not test if the pathway is active. Notch pathway activation can be visualized using tp1:eGFP Notch reporter fish ${ }^{29}$. At $36 \mathrm{hpf}$, mutant embryos show the same pattern of Notch signaling within the aorta and intersegmental vessels compared to their wild-type siblings (Figure 5D). These data indicate the lack of HSPC formation is not due to a lack of Notch signaling within arterial cells.

The results suggest that the hematopoietic defects in $s f 3 b 1$ mutants are likely due to problems transitioning between different cellular states, but cell death cannot be excluded as a contributing factor. We assessed apoptosis in $s f 3 b 1$ mutants using acridine orange staining and antibody staining for active caspase- $3^{41,42}$. While a clear increase in cell death in neuronal tissues, such as the brain and spinal cord, was observed, we noted no significant increase in apoptosis in other tissues in $s f 3 b 1$ mutants compared to wild-type siblings (Figure S4). These results support the model that splicing controls hematopoietic differentiation decisions.

\section{Discussion}

Recent clinical findings and discoveries in zebrafish development indicate that normal functioning of the spliceosome is critical for hematopoiesis. These studies also implied that the ensuing hematopoietic defects were somewhat distinct depending on the spliceosomal component affected. The most commonly mutated spliceosomal factor in MDS is SF3B1, yet there is currently no in vivo model describing what aspects of hematopoiesis are affected when this factor is lost. Here we demonstrated that depletion of $s f 3 b 1$ during zebrafish development results in deficits in primitive and definitive hematopoiesis. Primitive erythroid cells in sf $3 b 1$ mutants arise and initially differentiate normally, but then arrest at the orthochromatophilic stage and take on an abnormal megaloblastoid-like morphology. Primitive myelopoiesis also initiates normally, but then pu.1-expressing progenitors fail to mature. These data indicate $s f 3 b 1$ is needed for maturation of myeloid and erythroid progenitors. In contrast, induction of definitive hematopoiesis is severely impaired. Expression of the hemogenic endothelial and HSPC markers runx 1 and gata $2 b$ is hampered as early as $24 \mathrm{hpf}$ although the specification of non-hemogenic aortic endothelial cells at the same time and place in development appears mostly unaltered. These data suggest that $s f 3 b 1$ is differentially required in distinct endothelial cell populations and that hematopoiesis is acutely sensitive to perturbations in the spliceosome.

A common feature among the hematopoietic defects in $s f 3 b 1$ mutants is inhibited differentiation and maturation. Recent RNA-seq studies have revealed a complex orchestration of splicing throughout erythroid and myeloid differentiation ${ }^{53-57}$. The functional importance of these splicing events remains unclear. In erythroid maturation, 
splicing changes are greatest in the later stages of differentiation ${ }^{55,56}$. Our data provide a functional corollary to the importance of this observation as erythroid differentiation in sf $3 \mathrm{~b} 1$ mutants is blocked at a late progenitor stage. Unlike erythroid and myeloid splicing patterns, the splicing events occurring during the endothelial-to-hematopoietic transition are unknown. The lack of induction of expression of the key hemogenic transcription factors runx 1 and gata $2 b$ demonstrate splicing is a regulator of definitive HSPC origins. Further exploration of the splicing aberrations in $s f 3 b 1$ endothelial cells will help decipher which specific splice isoforms are important in HSPC development.

Although many spliceosomal mutants characterized to date have hematopoietic perturbations, they are not entirely overlapping, even in factors that are within the same complex. U2 snRNP is a large macromolecular complex made up of nearly 20 factors.

Zebrafish mutants of three of these factors ( $s f 3 a 3^{15}$, $d d x 46 / p r p 5^{12}$, and now $s f 3 b 1$ ) have been characterized, and all display defects in the hematopoietic system. Mutants in sf3a3 have normal primitive erythropoiesis and myelopoiesis, but show defects in HSPC formation, while $d d x 46$ mutants initiate HSPC induction normally, but have problems maintaining their stem cell pool. These studies lead to two non-mutually exclusive hypotheses for the differences observed: 1) different splicing events are affected in each spliceosomal mutants and 2) the diverse phenotypes could be due to non-spliceosomal functions of each splicing factor. Indeed, recent studies have found that $S F 3 B 1$ has some non-splicing functions in responding to various cellular stressors 58,59 .

These differences can also be observed clinically. MDS is a pleiotropic hematopoietic disease marked by perturbations to the erythroid and myeloid lineages. U2AF1 is an essential factor that associates with the U2 snRNP complex to help recruit it to splice sites. Despite the interconnection between these two complexes in splicing, mutations in $U 2 A F 1$ and $S F 3 B 1$ are associated with different features of MDS. Mutations in $U 2 A F 1$ segregate with MDS with poor overall survival, while mutations in $S F 3 B 1$ show a strong correlation with refractory anemia with ringed sideroblasts (RARS) and an overall favorable prognosis $^{4-10}$. Erythroid cells in RARS are poorly differentiated and show defects in iron usage resulting in hemoglobinization problems. Reminiscent of this type of MDS, zebrafish sf $3 \mathrm{~b} 1$ mutants have poorly differentiated red blood cells and low levels of functional hemoglobin, but they do not show symptoms of myeloproliferation associated with more malignant forms of MDS. Whether these differences are due to differences in splicing abnormalities or in non-splicing functions of U2AF1 and SF3B1 requires further exploration.

Combined, the clinical indications and differences in developmental hematopoietic deficiencies among spliceosomal mutants show that there is still much to learn about spliceosome protein function in metazoans. The zebrafish is an excellent model for addressing this problem. Maternal contribution of essential factors like spliceosomal components allows survival of embryos to later stages during organogenesis permitting comparison across many tissues. Zebrafish are highly amendable to genetic and chemical perturbations, which can be employed to define the functionally relevant pathways for each tissue type affected. Genome-wide studies of blood diseases, such as MDS, have revealed a number of correlations between gene mutation and disease phenotype, which are difficult to 
dissect due to the complexity of the mutational landscape within the heterogenous mix of cells analyzed. The use of in vivo systems, such as the zebrafish, provides a facile genetic approach for testing the contribution of single gene perturbations. We have now established numerous phenotypic similarities between zebrafish $s f 3 b 1$ mutants and $S F 3 B 1$-mutated MDS. Future studies will take advantage of this model to uncover the molecular and cellular origins of the hematopoietic defects arising from deficiencies in sf $3 b 1$.

\section{Supplementary Material}

Refer to Web version on PubMed Central for supplementary material.

\section{Acknowledgements}

This work was funded by Aplastic Anemia and MDS International Foundation (RCC), Training Program in Cellular and Molecular Biology and Genetics, T32 GM007491 (SN), Gabrielle's Angel Foundation, NIH 7K01DK085270-05, and NIH 6R03DK102975-02 (TVB).

We would like to thank Eirini Trompouki for critical reading of the work as well as Trista North, Leonard Zon and David Traver for sharing zebrafish strains and reagents.

\section{References}

1. Orkin SH, Zon LI. Hematopoiesis: an evolving paradigm for stem cell biology. Cell. 2008; 132:631644. doi:10.1016/j.cell.2008.01.025. [PubMed: 18295580]

2. Ellett F, Lieschke GJ. Zebrafish as a model for vertebrate hematopoiesis. Current opinion in pharmacology. 2010; 10:563-570. doi:10.1016/j.coph.2010.05.004. [PubMed: 20538521]

3. Clements WK, Traver D. Signalling pathways that control vertebrate haematopoietic stem cell specification. Nature reviews. Immunology. 2013; 13:336-348. doi:10.1038/nri3443.

4. Haferlach T, Nagata Y, Grossmann V, et al. Landscape of genetic lesions in 944 patients with myelodysplastic syndromes. Leukemia. 2014; 28:241-247. doi:10.1038/leu.2013.336. [PubMed: 24220272]

5. Hahn CN, Scott HS. Spliceosome mutations in hematopoietic malignancies. Nature genetics. 2012; 44:9-10. doi:10.1038/ng.1045. [PubMed: 22200771]

6. Papaemmanuil E, Cazzola M, Boultwood J, et al. Somatic SF3B1 mutation in myelodysplasia with ring sideroblasts. The New England journal of medicine. 2011; 365:1384-1395. doi:10.1056/ NEJMoa1 103283. [PubMed: 21995386]

7. Patnaik MM, Lasho TL, Hodnefield JM, et al. SF3B1 mutations are prevalent in myelodysplastic syndromes with ring sideroblasts but do not hold independent prognostic value. Blood. 2012; 119:569-572. doi:10.1182/blood-2011-09-377994. [PubMed: 22096241]

8. Yoshida K, Sanada M, Shiraishi Y, et al. Frequent pathway mutations of splicing machinery in myelodysplasia. Nature. 2011; 478:64-69. doi:10.1038/nature10496. [PubMed: 21909114]

9. Malcovati L, Karimi M, Papaemmanuil E, et al. SF3B1 mutation identifies a distinct subset of myelodysplastic syndrome with ring sideroblasts. Blood. 2015; 126:233-241. doi:10.1182/ blood-2015-03-633537. [PubMed: 25957392]

10. Makishima H, Visconte V, Sakaguchi H, et al. Mutations in the spliceosome machinery, a novel and ubiquitous pathway in leukemogenesis. Blood. 2012; 119:3203-3210. doi:10.1182/ blood-2011-12-399774. [PubMed: 22323480]

11. English MA, Lei L, Blake T, et al. Incomplete splicing, cell division defects, and hematopoietic blockage in dhx8 mutant zebrafish. Dev Dyn. 2012; 241:879-889. doi:10.1002/dvdy.23774. [PubMed: 22411201]

12. Hirabayashi R, Hozumi S, Higashijima S, Kikuchi Y. Ddx46 is required for multi-lineage differentiation of hematopoietic stem cells in zebrafish. Stem Cells Dev. 2013; 22:2532-2542. doi: 10.1089/scd.2012.0623. [PubMed: 23635340] 
13. Payne EM, Bolli N, Rhodes J, et al. Ddx18 is essential for cell-cycle progression in zebrafish hematopoietic cells and is mutated in human AML. Blood. 2011; 118:903-915. doi:10.1182/ blood-2010-11-318022. [PubMed: 21653321]

14. Keightley MC, Crowhurst MO, Layton JE, et al. In vivo mutation of pre-mRNA processing factor 8 (Prpf8) affects transcript splicing, cell survival and myeloid differentiation. FEBS Lett. 2013; 587:2150-2157. doi:10.1016/j.febslet.2013.05.030. [PubMed: 23714367]

15. Burns CE, Galloway JL, Smith AC, et al. A genetic screen in zebrafish defines a hierarchical network of pathways required for hematopoietic stem cell emergence. Blood. 2009; 113:57765782. doi:10.1182/blood-2008-12-193607. [PubMed: 19332767]

16. Danilova N, Kumagai A, Lin J. p53 upregulation is a frequent response to deficiency of cellessential genes. PloS one. 2010; 5:e15938. doi:10.1371/journal.pone.0015938. [PubMed: 21209837]

17. Isono K, Mizutani-Koseki Y, Komori T, Schmidt-Zachmann MS, Koseki H. Mammalian polycomb-mediated repression of Hox genes requires the essential spliceosomal protein Sf3b1. Genes Dev. 2005; 19:536-541. doi:10.1101/gad.1284605. [PubMed: 15741318]

18. Matsunawa M, Yamamoto R, Sanada M, et al. Haploinsufficiency of Sf3b1 leads to compromised stem cell function but not to myelodysplasia. Leukemia. 2014 doi:10.1038/leu.2014.73.

19. Wang C, Sashida G, Saraya A, et al. Depletion of Sf3b1 impairs proliferative capacity of hematopoietic stem cells but is not sufficient to induce myelodysplasia. Blood. 2014; 123:33363343. doi:10.1182/blood-2013-12-544544. [PubMed: 24735968]

20. Visconte V, Tabarroki A, Zhang L, et al. Splicing factor 3b subunit 1 (Sf3b1) haploinsufficient mice display features of low risk Myelodysplastic syndromes with ring sideroblasts. Journal of hematology \& oncology. 2014; 7:89. doi:10.1186/s13045-014-0089-x. [PubMed: 25481243]

21. An M, Henion PD. The zebrafish sf3b1b460 mutant reveals differential requirements for the sf3b1 pre-mRNA processing gene during neural crest development. Int J Dev Biol. 2012; 56:223-237. doi:10.1387/ijdb.113383ma. [PubMed: 22562198]

22. Amsterdam A, Nissen RM, Sun Z, et al. Identification of 315 genes essential for early zebrafish development. Proc Natl Acad Sci U S A. 2004; 101:12792-12797. doi:10.1073/pnas.0403929101. [PubMed: 15256591]

23. Lawrence C. Advances in zebrafish husbandry and management. Methods in cell biology. 2011; 104:429-451. doi:10.1016/B978-0-12-374814-0.00023-9. [PubMed: 21924176]

24. Lawrence CB,J, James A, Maloney K. The effects of feeding frequency on growth and reproduction in zebrafish (Danio rerio). Aquaculture. 2012; 368-369:103-108.

25. Long Q, Meng A, Wang H, et al. GATA-1 expression pattern can be recapitulated in living transgenic zebrafish using GFP reporter gene. Development. 1997; 124:4105-4111. [PubMed: 9374406]

26. Nakayama S, Ikenaga T, Kawakami K, Ono F, Hatta K. Transgenic line with gal4 insertion useful to study morphogenesis of craniofacial perichondrium, vascular endothelium-associated cells, floor plate, and dorsal midline radial glia during zebrafish development. Dev Growth Differ. 2012; 54:202-215. doi:10.1111/j.1440-169X.2011.01322.x. [PubMed: 22348745]

27. North TE, Goessling W, Walkley CR, et al. Prostaglandin E2 regulates vertebrate haematopoietic stem cell homeostasis. Nature. 2007; 447:1007-1011. doi:10.1038/nature05883. [PubMed: 17581586]

28. Hall C, Flores MV, Storm T, Crosier K, Crosier P. The zebrafish lysozyme C promoter drives myeloid-specific expression in transgenic fish. BMC Dev Biol. 2007; 7:42. doi: 10.1186/1471-213X-7-42. [PubMed: 17477879]

29. Parsons MJ, Pisharath H, Yusuff S, et al. Notch-responsive cells initiate the secondary transition in larval zebrafish pancreas. Mechanisms of development. 2009; 126:898-912. doi:10.1016/j.mod. 2009.07.002. [PubMed: 19595765]

30. Thisse C, Thisse B. High-resolution in situ hybridization to whole-mount zebrafish embryos. Nat Protoc. 2008; 3:59-69. doi:10.1038/nprot.2007.514. [PubMed: 18193022]

31. Brownlie A, Hersey C, Oates AC, et al. Characterization of embryonic globin genes of the zebrafish. Dev Biol. 2003; 255:48-61. [PubMed: 12618133] 
32. Lawson ND, Scheer N, Pham VN, et al. Notch signaling is required for arterial-venous differentiation during embryonic vascular development. Development. 2001; 128:3675-3683. [PubMed: 11585794]

33. Thompson MA, Ransom DG, Pratt SJ, et al. The cloche and spadetail genes differentially affect hematopoiesis and vasculogenesis. Dev Biol. 1998; 197:248-269. doi:10.1006/dbio.1998.8887. [PubMed: 9630750]

34. Herbomel P, Thisse B, Thisse C. Ontogeny and behaviour of early macrophages in the zebrafish embryo. Development. 1999; 126:3735-3745. [PubMed: 10433904]

35. Lieschke GJ, Oates AC, Crowhurst MO, Ward AC, Layton JE. Morphologic and functional characterization of granulocytes and macrophages in embryonic and adult zebrafish. Blood. 2001; 98:3087-3096. [PubMed: 11698295]

36. Lieschke GJ, Oates AC, Paw BH, et al. Zebrafish SPI-1 (PU.1) marks a site of myeloid development independent of primitive erythropoiesis: implications for axial patterning. Dev Biol. 2002; 246:274-295. doi:10.1006/dbio.2002.0657. [PubMed: 12051816]

37. Burns CE, DeBlasio T, Zhou Y, et al. Isolation and characterization of runxa and runxb, zebrafish members of the runt family of transcriptional regulators. Exp Hematol. 2002; 30:1381-1389. [PubMed: 12482499]

38. Butko E, Distel M, Pouget C, et al. Gata2b is a restricted early regulator of hemogenic endothelium in the zebrafish embryo. Development. 2015; 142:1050-1061. doi:10.1242/dev.119180. [PubMed: 25758220]

39. Liao EC, Paw BH, Oates AC, et al. SCL/Tal-1 transcription factor acts downstream of cloche to specify hematopoietic and vascular progenitors in zebrafish. Genes Dev. 1998; 12:621-626. [PubMed: 9499398]

40. Renaud O, Herbomel P, Kissa K. Studying cell behavior in whole zebrafish embryos by confocal live imaging: application to hematopoietic stem cells. Nat Protoc. 2011; 6:1897-1904. doi: 10.1038/nprot.2011.408. [PubMed: 22082984]

41. Tucker B, Lardelli M. A rapid apoptosis assay measuring relative acridine orange fluorescence in zebrafish embryos. Zebrafish. 2007; 4:113-116. doi:10.1089/zeb.2007.0508. [PubMed: 18041929]

42. Sorrells S, Toruno C, Stewart RA, Jette C. Analysis of apoptosis in zebrafish embryos by wholemount immunofluorescence to detect activated Caspase 3. Journal of visualized experiments: JoVE. 2013:e51060. doi:10.3791/51060. [PubMed: 24378359]

43. North TE, Goessling W, Peeters M, et al. Hematopoietic stem cell development is dependent on blood flow. Cell. 2009; 137:736-748. doi:10.1016/j.cell.2009.04.023. [PubMed: 19450519]

44. Lyons SE, Lawson ND, Lei L, et al. A nonsense mutation in zebrafish gata1 causes the bloodless phenotype in vlad tepes. Proc Natl Acad Sci U S A. 2002; 99:5454-5459. doi:10.1073/pnas. 082695299. [PubMed: 11960002]

45. Brownlie A, Donovan A, Pratt SJ, et al. Positional cloning of the zebrafish sauternes gene: a model for congenital sideroblastic anaemia. Nat Genet. 1998; 20:244-250. doi:10.1038/3049. [PubMed: 9806542]

46. Songhet P, Adzic D, Reibe S, Rohr KB. fgf1 is required for normal differentiation of erythrocytes in zebrafish primitive hematopoiesis. Dev Dyn. 2007; 236:633-643. doi:10.1002/dvdy.21056. [PubMed: 17219402]

47. Paffett-Lugassy NN, Zon LI. Analysis of hematopoietic development in the zebrafish. Methods in molecular medicine. 2005; 105:171-198. [PubMed: 15492396]

48. Bertrand JY, Cisson JL, Stachura DL, Traver D. Notch signaling distinguishes 2 waves of definitive hematopoiesis in the zebrafish embryo. Blood. 2010; 115:2777-2783. doi:10.1182/ blood-2009-09-244590. [PubMed: 20107232]

49. Bertrand JY, Kim AD, Violette EP, et al. Definitive hematopoiesis initiates through a committed erythromyeloid progenitor in the zebrafish embryo. Development. 2007; 134:4147-4156. doi: 10.1242/dev.012385. [PubMed: 17959717]

50. Ciau-Uitz A, Monteiro R, Kirmizitas A, Patient R. Developmental hematopoiesis: ontogeny, genetic programming and conservation. Exp Hematol. 2014; 42:669-683. doi:10.1016/j.exphem. 2014.06.001. [PubMed: 24950425] 
51. Wang L, Zhang P, Wei Y, et al. A blood flow-dependent klf2a-NO signaling cascade is required for stabilization of hematopoietic stem cell programming in zebrafish embryos. Blood. 2011; 118:4102-4110. doi:10.1182/blood-2011-05-353235. [PubMed: 21849483]

52. Bertrand JY, Chi NC, Santoso B, et al. Haematopoietic stem cells derive directly from aortic endothelium during development. Nature. 2010; 464:108-111. doi:10.1038/nature08738. [PubMed: 20154733]

53. Bhatt DM, Pandya-Jones A, Tong AJ, et al. Transcript dynamics of proinflammatory genes revealed by sequence analysis of subcellular RNA fractions. Cell. 2012; 150:279-290. doi: 10.1016/j.cell.2012.05.043. [PubMed: 22817891]

54. Wong JJ, Ritchie W, Ebner OA, et al. Orchestrated intron retention regulates normal granulocyte differentiation. Cell. 2013; 154:583-595. doi:10.1016/j.cell.2013.06.052. [PubMed: 23911323]

55. Pimentel H, Parra M, Gee SL, et al. A dynamic intron retention program enriched in RNA processing genes regulates gene expression during terminal erythropoiesis. Nucleic acids research. 2015 doi:10.1093/nar/gkv1168.

56. Pimentel H, Parra M, Gee S, et al. A dynamic alternative splicing program regulates gene expression during terminal erythropoiesis. Nucleic acids research. 2014; 42:4031-4042. doi: 10.1093/nar/gkt1388. [PubMed: 24442673]

57. Yamamoto ML, Clark TA, Gee SL, et al. Alternative pre-mRNA splicing switches modulate gene expression in late erythropoiesis. Blood. 2009; 113:3363-3370. doi:10.1182/ blood-2008-05-160325. [PubMed: 19196664]

58. Schreiber CA, Sakuma T, Izumiya Y, et al. An siRNA Screen Identifies the U2 snRNP Spliceosome as a Host Restriction Factor for Recombinant Adeno-associated Viruses. PLoS Pathog. 2015; 11:e1005082. doi:10.1371/journal.ppat.1005082. [PubMed: 26244496]

59. Paulsen RD, Soni DV, Wollman R, et al. A genome-wide siRNA screen reveals diverse cellular processes and pathways that mediate genome stability. Molecular cell. 2009; 35:228-239. doi: 10.1016/j.molcel.2009.06.021. [PubMed: 19647519] 


\section{Highlights}

- $\quad$ Erythroid and myeloid differentiation is inhibited in $s f 3 b 1$ zebrafish mutants.

- $\quad \mathrm{Sf3b1}$ is required for hematopoietic stem and progenitor cell emergence (HSPC).

- $\quad$ Loss of HSPCs is independent to defects in endothelial cell specification.

- $\quad$ Notch signaling in the aorta does not require Sf3b1. 
A.

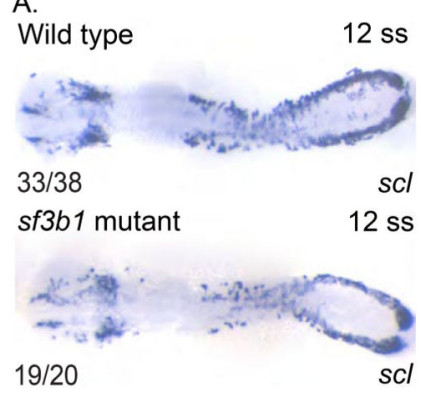

D.
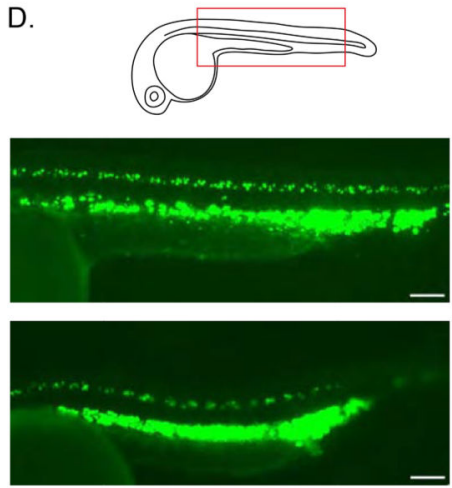

G. Wild type

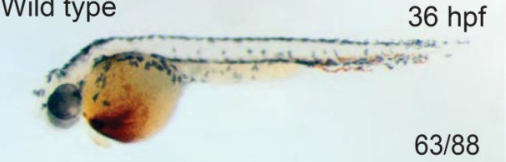

sf3b1 mutant

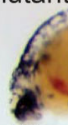

o-dianisidine

36 hpf

$35 / 47$

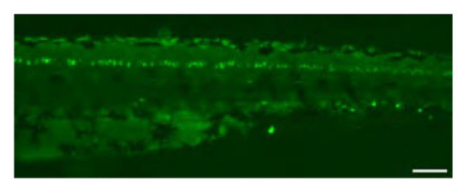

E.
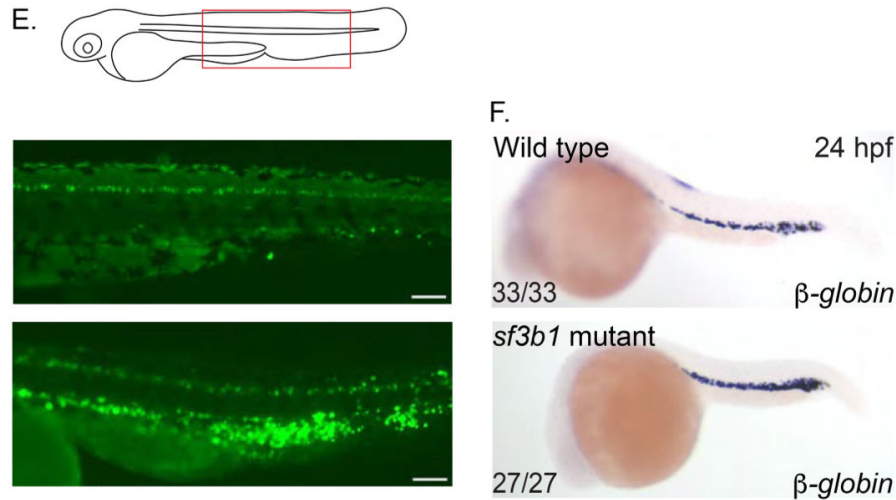

C.

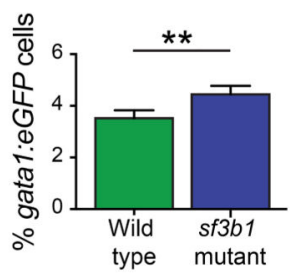

H.

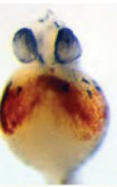

Wild type

$48 \mathrm{hpf}$

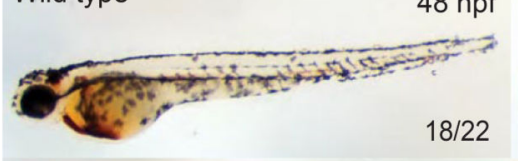

sf3b1 mutant

$48 \mathrm{hpf}$

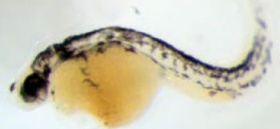

$28 / 39$
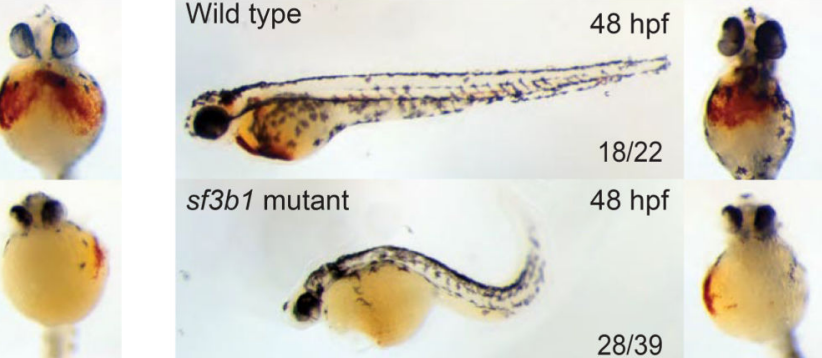

I.

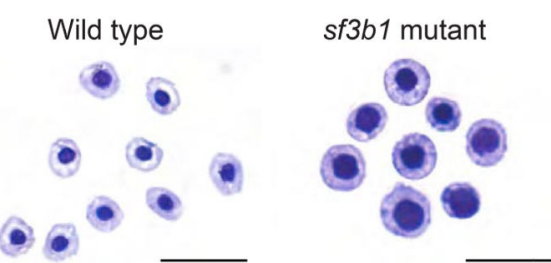

J.
\begin{tabular}{|l|c|c|c|}
\hline & Proerythroblast & $\begin{array}{c}\text { Basophilic } \\
\text { erythroblast }\end{array}$ & $\begin{array}{c}\text { Orthochromatophilic } \\
\text { erythroblast }\end{array}$ \\
\hline Wildtype & $6(2 \%)$ & $30(11 \%)$ & $241(87 \%)$ \\
\hline Mutants & $37(23 \%)$ & $35(22 \%)$ & $86(55 \%)$ \\
\hline
\end{tabular}

Figure 1. $s f 3 b 1$ mutants have normal erythroid cell development but lack mature erythrocytes A. In situ hybridization of the primitive hematopoietic marker $s c l$ at 12 ss (14 hpf) in wild type (top) and $s f 3 b 1$ mutants (bottom). Number shown in the lower left corner denotes the number of embryos of each genotype that displayed a similar phenotype to the image. B. Flow cytometry analysis of gata1:eGFP cells from wild type (left) and $s f 3 b 1$ mutants (right). Averages +/- standard deviation of 3 replicates are shown. For each replicate, 10-20 embryos were pooled for the analysis. C. Graph showing the percentage of gata1:eGFP positive cells shown in B. Student's t-test $* * p=0.007$. D,E. Schematic showing area of the 
embryo shown in images below. Fluorescent images of gata1:eGFP wild type (top) and sf3b1 mutants (bottom) at $24 \mathrm{hpf}$ (D.) and $48 \mathrm{hpf}$ (E.), scale bar: $100 \mu \mathrm{m}$. F. In situ hybridization of the erythroid marker $\beta$-globin at $24 \mathrm{hpf}$ in wild type (top) and sf3b1 mutants (bottom). G,H. O-dianisidine staining of functional hemoglobin in mature primitive erythrocytes in wild type (top) and $s f 3 b 1$ mutants (bottom) at $36 \mathrm{hpf}$ (G.) and $48 \mathrm{hpf}$ (H.). I. Representative images of orthochromatophilic erythroblasts stained with May GrunwaldGiemsa from wild type (left) and $s f 3 b 1$ mutants (right), scale bar: $20 \mu \mathrm{m}$. J. Number of cells that were classified by morphology at each differentiation stage with percentages shown in brackets for wild type and $s f 3 b 1$ mutants. Chi-squared test $* * * * \mathrm{p}=0.0001$. 
A.

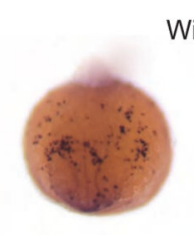

22 hpf

B.

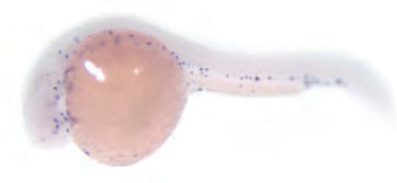

$24 \mathrm{hpf}$

47/47 I-plastin

C.

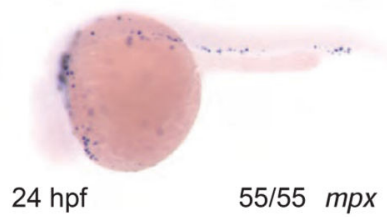

D.
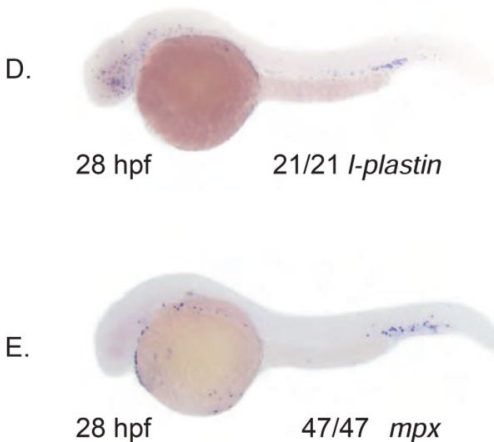

F.

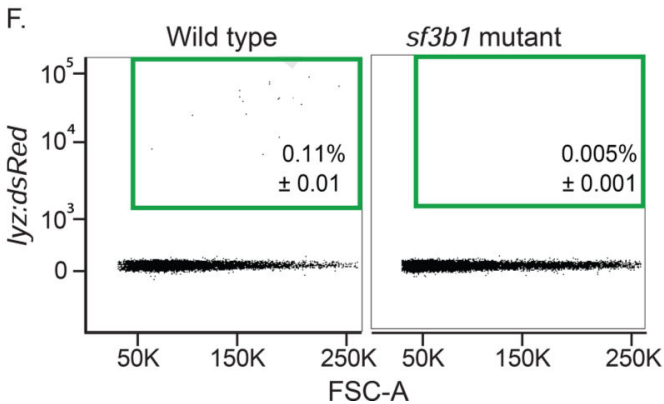

sf3b1 mutant

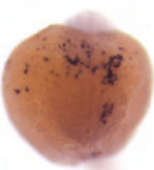

22 hpf

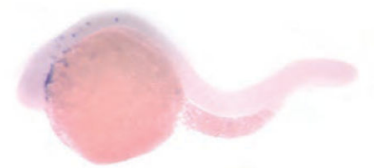

24 hpf

26/26 I-plastin

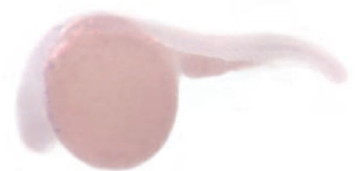

24 hpf

$31 / 31 m p x$
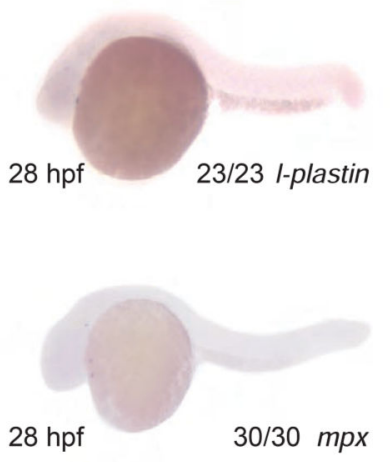

G.

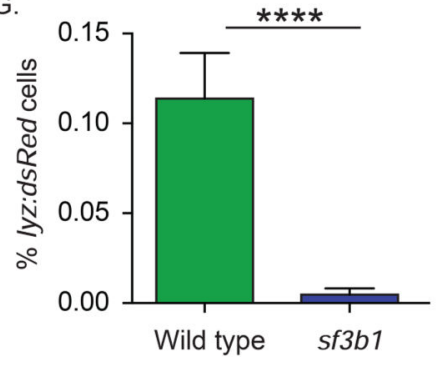

Figure 2. Primitive myeloid cell development is impaired in $s f 3 b 1$ mutants

A-E. In situ hybridization for myeloid markers. Images of wild type are shown on the left and $s f 3 b 1$ mutants are shown on the right. Number shown in the lower right corner denotes the number of embryos of each genotype that displayed a similar phenotype to the image. A. The myeloid progenitor marker pu. 1 at $22 \mathrm{hpf}$. B,D. The myeloid differentiation marker 1 plastin at $24 \mathrm{hpf}$ (B.) and $28 \mathrm{hpf}$ (D.) C,E. The neutrophil marker mpx at $24 \mathrm{hpf}$ (C.) and 28 hpf (E.). F. Flow cytometry analysis of lyz:dsRed-positive myeloid cells from wild type (left) and $s f 3 b 1$ mutants (right) at $36 \mathrm{hpf}$. Averages $+/-$ standard deviation of 3 replicates are 
shown. For each replicate, 10-20 embryos were pooled for the analysis. G. Graph showing the percentage of lyz.dsRed positive cells shown in F. Student's t-test $* * * * p=0.0001$. 
Wild type

A.

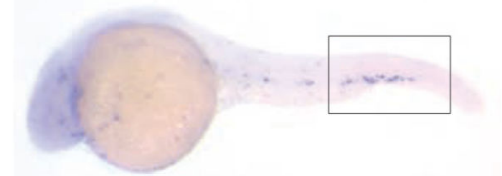

26 hpf $25 / 25$ pu. 1

B.

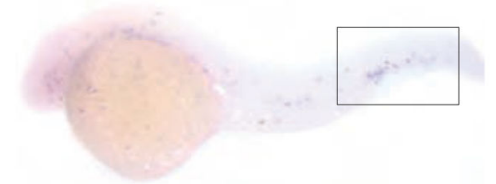

$30 \mathrm{hpf}$ 23/23 I-plastin

C.

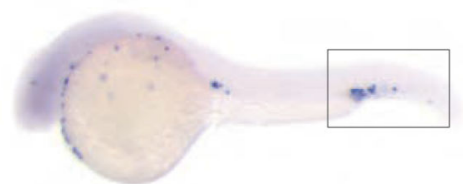

$30 \mathrm{hpf}$
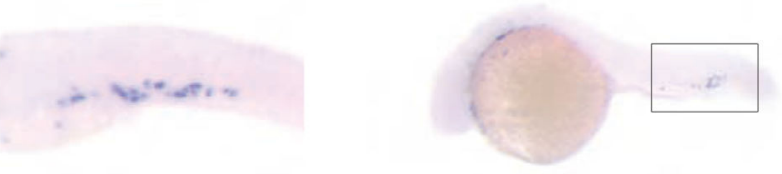

$26 \mathrm{hpf}$

$19 / 19$ pu. 1
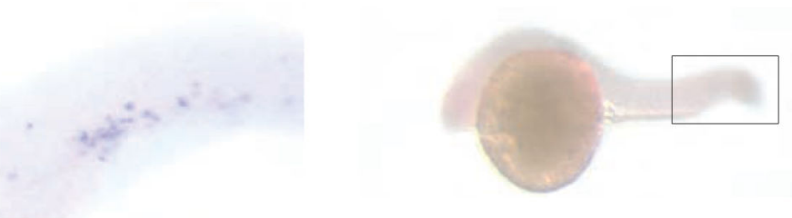

$30 \mathrm{hpf} \quad 24 / 24$ I-plastin

Figure 3. Erythroid-myeloid progenitor development is impaired in $s f 3 b 1$ mutants

A-C. In situ hybridization for myeloid markers. Images of wild type are shown on the left and $s f 3 b 1$ mutants are shown on the right. Number shown in the lower right corner denotes the number of embryos of each genotype that displayed a similar phenotype to the image. Inset to the right shows a higher magnification view of the PBI region of the embryo boxed in the image on the left. A. The myeloid progenitor marker pu. 1 at $26 \mathrm{hpf}$. B. The myeloid differentiation marker 1-plastin at $30 \mathrm{hpf}$. C. The neutrophil marker $m p x$ at $30 \mathrm{hpf}$. 
A.

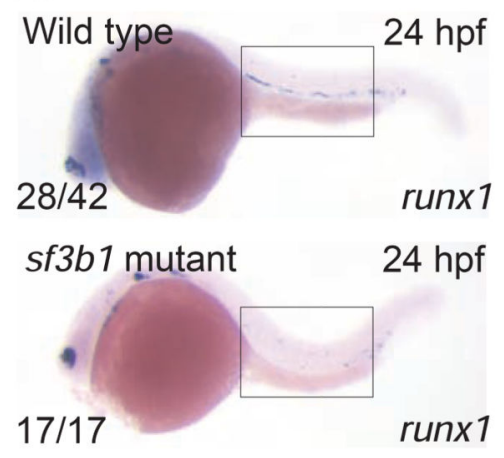

C.
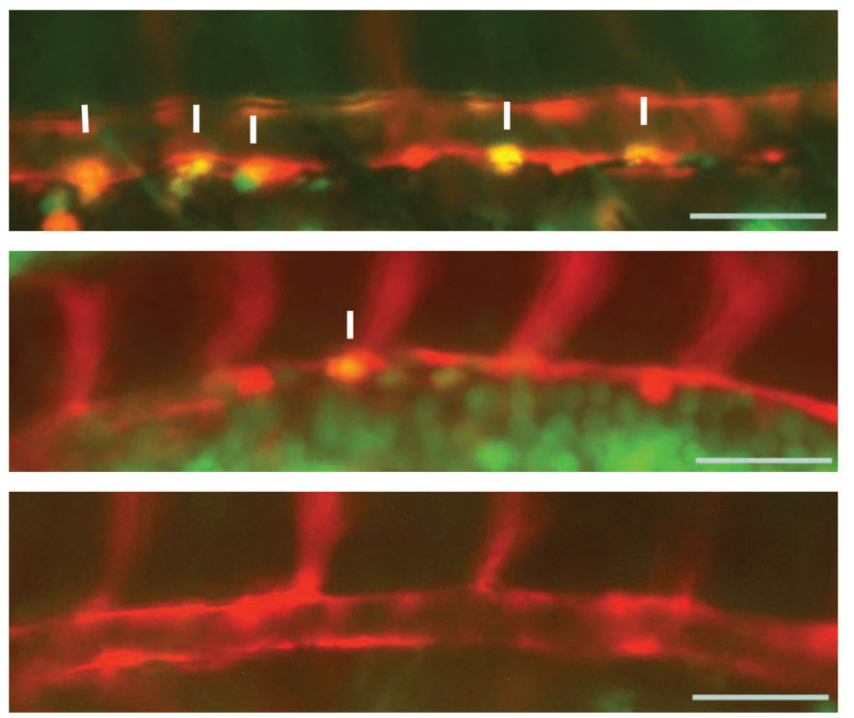

B.
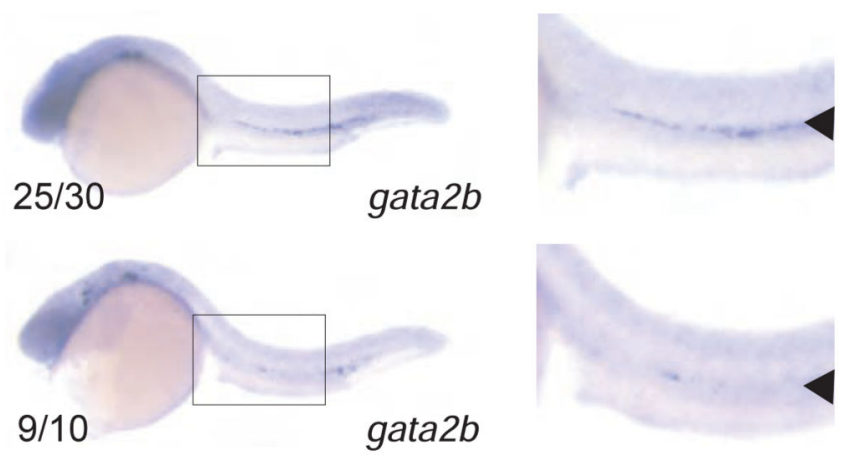

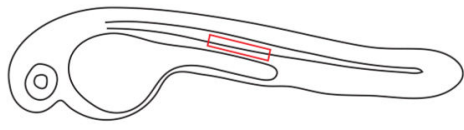

D.

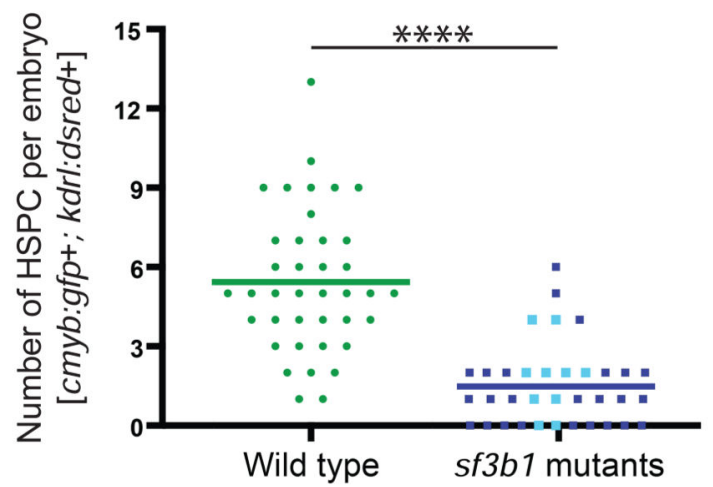

Figure 4. sf3b1 mutants have diminished HSPCs

A,B. In situ hybridization of the HSPC markers runx 1 (A.) and gata2b (B.) at $24 \mathrm{hpf}$ in wild type (top) and $s f 3 b 1$ mutants (bottom). Inset to the right shows a higher magnification view of the AGM region of the embryo boxed in the image on the left with aorta marked with arrowheads. Number shown in the lower left corner denotes the number of embryos of each genotype that displayed a similar phenotype to the image. C. Representative fluorescent images of $c m y b$ :gfp+; $k d r l:$ dsred+ HSPCs (arrows) from wild type (top) and sf3b1 mutants (bottom) at $38 \mathrm{hpf}$. Middle image depicts a mutant with a less inflated aorta and the bottom image shows a mutant with a more inflated aorta. Scale bar: 50 $\mu \mathrm{M}$. Schematic on the right shows the area of the embryo shown in the fluorescent images. D. Graph showing quantification of HSPCs ( $c m y b$ :gfp+; $k d r l:$ dsred+ cells) in wild type and $s f 3 b 1$ mutants at 38 hpf. Each dot represents the number of HSPC per embryo and the line denotes the mean. Dark blue dots are HSPC counts for mutants with less inflated aorta and the light blue dots are HSPC counts for mutants with more inflated aorta. Student's t-test **** $\mathrm{p}=6 \times 10^{-11}$. 
Wild type

A.

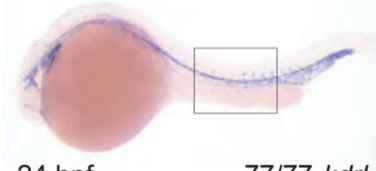

$24 \mathrm{hpf}$

B.

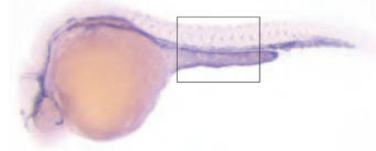

$24 \mathrm{hpf}$

C.

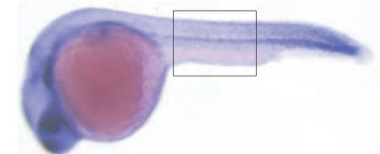

$24 \mathrm{hpf}$

28/28 ephb2

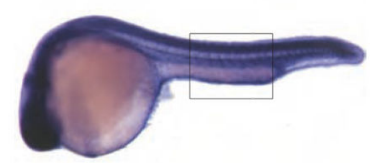

$24 \mathrm{hpf}$

38/38 notch $1 b$

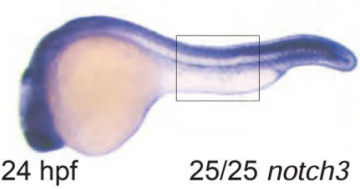

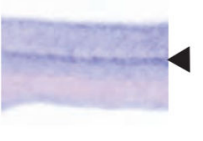
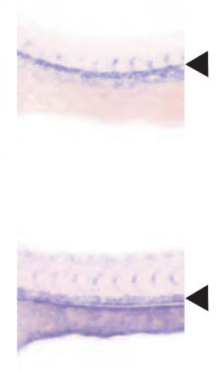

$23 / 24$ flt 4
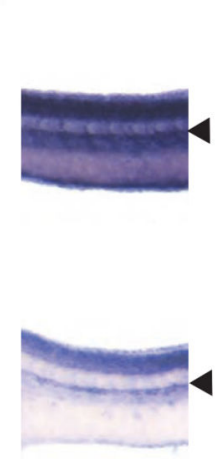

sf $3 b 1$ mutant

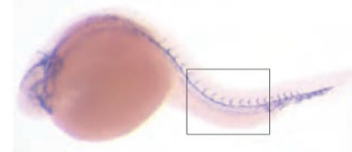

$24 \mathrm{hpf}$

$36 / 36 k d r l$

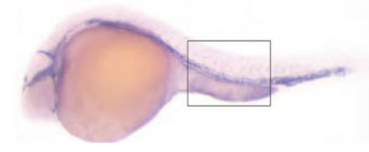

$24 \mathrm{hpf}$

$16 / 16$ flt4
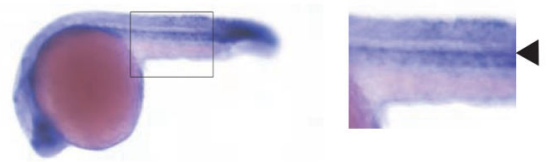

$24 \mathrm{hpf}$

21/21 ephb2

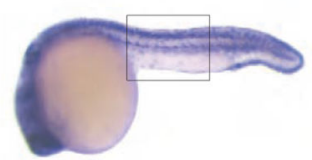

$24 \mathrm{hpf}$

26/26 notch $1 b$
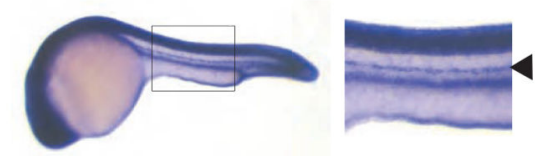

$24 \mathrm{hpf}$

18/18 notch3

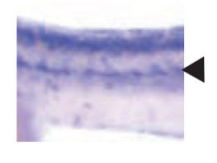

D.

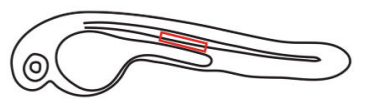

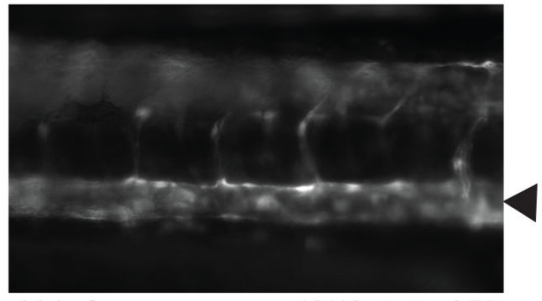

$36 \mathrm{hpf}$

13/13 tp1:eGFP

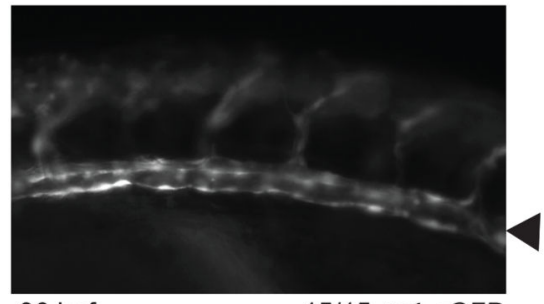

$36 \mathrm{hpf}$
15/15 tp1:eGFP

Figure 5. Endothelial markers are expressed in the correct vessels in $s f 3 b 1$ mutants

A-C. In situ hybridization for vascular markers at $24 \mathrm{hpf}$. Images of wild type are shown on the left and $s f 3 b 1$ mutants are shown on the right. Number shown in the lower right corner denotes the number of embryos of each genotype that displayed a similar phenotype to the image. Inset to the right shows a higher magnification view of the AGM region of the embryo boxed in the image on the left with aorta or vein marked with arrowheads. A. The pan-endothelial marker $k d r l$. B. The vein marker flt4. C. The aorta-specific markers ephrinb2, notch $1 b$ and notch3. D. Schematic showing area of the embryo shown in images 
below. Representative fluorescent images of $t p 1$ eGFP (Notch reporter) wild type (left) and sf $3 b 1$ mutants (right) at $36 \mathrm{hpf}$. Arrowhead denotes the aorta. 